\title{
$\varnothing$ EDITOR'S CHOICE \\ 2010 Rheumatoid arthritis classification criteria: an American College of Rheumatology/European League Against Rheumatism collaborative initiative
}

\author{
Daniel Aletaha, ${ }^{1}$ Tuhina Neogi, ${ }^{2}$ Alan J Silman, ${ }^{3}$ Julia Funovits, ${ }^{1}$ David T Felson, ${ }^{2}$ \\ Clifton 0 Bingham III, ${ }^{4}$ Neal S Birnbaum, ${ }^{5}$ Gerd R Burmester, ${ }^{6}$ Vivian P Bykerk, ${ }^{7}$ \\ Marc D Cohen, ${ }^{8}$ Bernard Combe, ${ }^{9}$ Karen H Costenbader, ${ }^{10}$ Maxime Dougados, ${ }^{11}$ \\ Paul Emery, ${ }^{12}$ Gianfranco Ferraccioli, ${ }^{13}$ Johanna MW Hazes, ${ }^{14}$ Kathryn Hobbs, ${ }^{15}$ \\ Tom WJ Huizinga, ${ }^{16}$ Arthur Kavanaugh, ${ }^{17}$ Jonathan Kay, ${ }^{18}$ Tore K Kvien, ${ }^{19}$ \\ Timothy Laing, ${ }^{20}$ Philip Mease, ${ }^{21}$ Henri A Ménard, ${ }^{22}$ Larry W Moreland, ${ }^{23}$ \\ Raymond L Naden, ${ }^{24}$ Theodore Pincus, ${ }^{25}$ Josef S Smolen, ${ }^{1}$ Ewa Stanislawska-Biernat, ${ }^{26}$ \\ Deborah Symmons, ${ }^{27}$ Paul P Tak, ${ }^{28}$ Katherine S Upchurch, ${ }^{18}$ Jiří Vencovský, ${ }^{29}$ \\ Frederick Wolfe, ${ }^{30}$ Gillian Hawker, ${ }^{31}$
}

For numbered affiliations see end of article

\section{Correspondence to Dr Alan J Silman, Arthritis Research UK, Copeman House, Chesterfield S41 7TD, UK: a.silman@arthritisresearchuk. org}

Accepted 28 June 2010

This article is published simultaneously in the September 2010 issue of Arthritis \& Rheumatism.

Supported by the American College of Rheumatology and the European League Against Rheumatism.

\section{ABSTRACT}

Objective The 1987 American College of Rheumatology (ACR; formerly the American Rheumatism Association) classification criteria for rheumatoid arthritis (RA) have been criticised for their lack of sensitivity in early disease. This work was undertaken to develop new classification criteria for RA.

Methods A joint working group from the ACR and the European League Against Rheumatism developed, in three phases, a new approach to classifying RA. The work focused on identifying, among patients newly presenting with undifferentiated inflammatory synovitis, factors that best discriminated between those who were and those who were not at high risk for persistent and/ or erosive disease- - this being the appropriate current paradigm underlying the disease construct ' $R A$ '.

Results In the new criteria set, classification as 'definite $R A^{\prime}$ is based on the confirmed presence of synovitis in at least one joint, absence of an alternative diagnosis better explaining the synovitis, and achievement of a total score of 6 or greater (of a possible 10) from the individual scores in four domains: number and site of involved joints (range $0-5$ ), serological abnormality (range $0-3$ ), elevated acute-phase response (range $0-1$ ) and symptom duration (two levels; range 0-1).

Conclusion This new classification system redefines the current paradigm of RA by focusing on features at earlier stages of disease that are associated with persistent and/or erosive disease, rather than defining the disease by its late-stage features. This will refocus attention on the important need for earlier diagnosis and institution of effective disease-suppressing therapy to prevent or minimise the occurrence of the undesirable sequelae that currently comprise the paradigm underlying the disease construct 'RA'.

Rheumatoid arthritis (RA) is a chronic inflammatory disease characterised by joint swelling, joint tenderness and destruction of synovial joints, leading to severe disability and premature mortality. ${ }^{1-5}$ Given the presence of autoantibodies, such as rheumatoid factor (RF) and anti-citrullinated protein
- This criteria set has been approved by the American College of Rheumatology (ACR) Board of Directors and the European League Against Rheumatism (EULAR) Executive Committee.

- This signifies that the criteria set has been quantitatively validated using patient data, and it has undergone validation based on an external data set.

- All ACR/EULARapproved criteria sets are expected to undergo intermittent updates

- The American College of Rheumatology is an independent, professional, medical and scientific society which does not guarantee, warrant, or endorse any commercial product or service.

antibody (ACPA) (tested as anti-cyclic citrullinated peptide), which can precede the clinical manifestation of RA by many years, ${ }^{6-9}$ RA is considered an autoimmune disease. ${ }^{10} 11$ Autoimmunity and the overall systemic and articular inflammatory load drive the destructive progression of the disease. However, although structural changes, which can be visualised by conventional radiography or other imaging techniques, best distinguish RA from other arthritic disorders, ${ }^{12}$ joint damage is rarely apparent in the very early stages of disease, but rather accumulates consistently over time. ${ }^{13-16}$

Over the last decade, the optimal use of diseasemodifying antirheumatic drugs (DMARD), in particular the anchor DMARD methotrexate, ${ }^{17-19}$ and the availability of new biological agents, ${ }^{11} 20$ have dramatically enhanced the success of RA management. Moreover, it has been recognised that early therapeutic intervention improves clinical outcomes and reduces the accrual of joint damage and disability. ${ }^{21-23}$ Undoubtedly, treating patients at a stage at which evolution of joint destruction can still be prevented would be ideal. However, at 
present, clinical trials of RA treatments are hampered by lack of criteria allowing for study enrolment of patients at early stages of disease. Thus, to date it has not been possible effectively to investigate the efficacy of early interventions in terms of their ability to prevent later-stage RA, since there are no validated or accepted uniform criteria to classify such individuals with early disease.

The standard and accepted means of defining RA is by use of classification criteria. Classification criteria enable the stratification of groups of individuals into those with and those without $\mathrm{RA}$ in order to standardise recruitment into clinical trials and related studies, and provide the basis for a common approach to disease definition that can be used to compare across studies and centres. The classification criteria set that is in widespread international use to define RA are the 1987 American College of Rheumatology (ACR; formerly the American Rheumatism Association) criteria. ${ }^{24}$ These criteria are well accepted as providing the benchmark for disease definition, but have a significant limitation in that they were derived by trying to discriminate patients with established RA from those with a combination of other definite rheumatological diagnoses. They are therefore not helpful in achieving the goal of identifying patients who would benefit from early effective intervention, as discussed above. Indeed, with modern therapies, the goal is to prevent individuals from reaching the chronic, erosive disease state that is exemplified in the 1987 criteria for RA.

A joint working group of the ACR and the European League Against Rheumatism (EULAR) was therefore formed to develop a new approach for classification of RA. While classification criteria are potentially adopted for use as aids for diagnosis, the focus of this endeavour was not on developing diagnostic criteria or providing a referral tool for primary care physicians. Indeed, a separate body of work is needed to develop such tools, which may be informed by classification criteria. Thus, the specific charge was to develop new classification criteria for RA to facilitate the study of persons at earlier stages of the disease. It was with this framework in mind that the working group developed the 2010 ACR/EULAR classification criteria for RA.

\section{OVERVIEW ON HYPOTHESIS AND METHODS OF PHASES 1 AND 2}

A priori, the working group focused on developing an approach that would be appropriate for newly presenting patients with undifferentiated inflammatory synovitis, in order to identify that subset of patients who are at sufficiently high risk of persistent and/or erosive disease-this being the appropriate current paradigm underlying the disease construct ' $\mathrm{RA}$ '- to be classified as having RA. It was recognised that such a scheme should not be developed using existing criteria sets as the 'gold standard,' because of the inherent circularity. The goal set forth was to develop a set of rules to be applied to newly presenting patients with undifferentiated synovitis that would: (1) identify the subset at high risk of chronicity and erosive damage; (2) be used as a basis for initiating disease-modifying therapy; and (3) not exclude the capture of patients later in the disease course.

To achieve these goals, the working group devised a three phase programme. Phase 1 was a data-driven approach based on cohorts of real-world patients with early arthritis, to identify factors and their relative weights, which were associated with the subsequent decision by a physician to start methotrexate treatment. Phase 2 was a consensus-driven, decision sciencebased approach, informed by the data from Phase 1 , to refine these factors and their weights using a series of 'paper patients', as well as to identify any other factors that may be of relevance based on current clinical thinking. Phase 3, which is the focus of this report, describes the derivation, from the previous two phases, of the final classification criteria set. The details of the methods and results from Phases 1 and 2 are provided elsewhere, ${ }^{25} 26$ and are briefly summarised below.

\section{Phase 1}

The aim of Phase 1 was to identify the contributions of clini$\mathrm{cal}$ and laboratory variables that in practice were the most predictive of the decision to initiate DMARD therapy in a population of patients with early undifferentiated synovitis. Initiation of DMARD therapy was used as an indicator of the physician's opinion that the patient was at risk of developing persistent and/or erosive arthritis that we would currently consider to be RA. Data on 3115 patients from nine early arthritis cohorts who were considered not to have evidence of another possible diagnosis explaining their presentation were obtained. Between July 2007 and November 2008 an expert working group developed an analysis strategy that related an agreed-upon list of standardised clinical and laboratory variables collected at baseline to the initiation of DMARD treatment within the next 12 months. Methotrexate initiation was used as the gold standard for this purpose. The analytical process aimed to identify the independent contribution of each variable on this list and included univariate regression modelling, a subsequent principal components analysis, and a multivariate regression model that included all identified components. ${ }^{25}$ The resulting list of informative variables identified during that process and the weights based on the odds ratios are shown in table 1.

\section{Phase 2}

Phase 2 consisted of a consensus-based, decision scienceinformed approach, which took place between November 2008 and June 2009. The purpose of this phase was to derive a clinician-based judgement on the relative contribution of clinical and laboratory factors deemed to be important in influencing the probability of developing 'persistent inflammatory and/or erosive arthritis that is currently considered to be RA' (hereinafter referred to as 'developing RA').

An expert panel was assembled, comprising 12 rheumatologists from Europe and 12 from North America with extensive experience in the diagnosis and management of RA. They provided real-life case scenarios of patients with early undifferentiated inflammatory arthritis representing low to high

Table 1 Summary of Phase 1 results

\begin{tabular}{llc}
\hline Variable & Comparison & Relative weight† \\
\hline Swollen MCP joint & Present versus absent & 1.5 \\
Swollen PIP joint & Present versus absent & 1.5 \\
Swollen wrist & Present versus absent & 1.6 \\
Hand tenderness & Present versus absent & 1.8 \\
Acute-phase response & Lowly abnormal versus normal & 1.2 \\
& Highly abnormal versus normal & 1.7 \\
Serology (RF or ACPA) & Low-positive versus negative & 2.2 \\
& High-positive versus negative & 3.9 \\
\hline
\end{tabular}

tDerived from OR from the multivariate regression model, and interpreted as the increase in the odds of having RA with as opposed to without the respective feature (eg, weight of 1.5 for swelling of PIP joints means that the odds of having RA is 1.5-fold in patients with as opposed to patients without swelling of a PIP joint).

ACPA, anti-citrullinated protein antibody; MCP, metacarpophalangeal; PIP, proximal interphalangeal; RA, rheumatoid arthritis; $\mathrm{RF}$, rheumatoid factor. 
Table 2 Summary of Phase 2 results and subsequent modifications

\begin{tabular}{|c|c|c|c|}
\hline & Exact $(0-100)$ & Rescaled $(0-10)$ & Rounded to $0.5(0-10)$ \\
\hline \multicolumn{4}{|l|}{ Joint involvement* ${ }^{*}$} \\
\hline 1 large & 0 & 0 & 0 \\
\hline$>1-10$ large, asymmetric & 10.2 & 1.02 & 1 \\
\hline$>1-10$ large, symmetric & 16.1 & 1.61 & 1.5 \\
\hline $1-3$ small & 21.2 & 2.12 & 2 \\
\hline $4-10$ small & 28.8 & 2.88 & 3 \\
\hline$>10$, including at least 1 small joint & 50.8 & 5.08 & 5 \\
\hline \multicolumn{4}{|l|}{ Serologyt } \\
\hline Negative RF and negative ACPA & 0 & 0 & 0 \\
\hline Low-positive RF or low-positive ACPA & 22.0 & 2.20 & 2 \\
\hline High-positive RF or high-positive ACPA & 33.9 & 3.39 & 3.5 \\
\hline \multicolumn{4}{|l|}{ Acute-phase reactants $\ddagger$} \\
\hline Normal CRP and normal ESR & 0 & 0 & 0 \\
\hline Abnormal CRP or abnormal ESR & 5.9 & 0.59 & 0.5 \\
\hline \multicolumn{4}{|l|}{ Duration of symptoms§ } \\
\hline$<6$ weeks & 0 & 0 & 0 \\
\hline$\geq 6$ weeks & 9.3 & 0.93 & 1 \\
\hline \multicolumn{4}{|c|}{$\begin{array}{l}\text { *Joint involvement refers to any swollen or tender joint on examination. Distal interphalangeal joints, first carpometacarpal joints } \\
\text { and first metatarsophalangeal (MTP) joints are excluded from assessment. Categories of joint distribution are classified according } \\
\text { to the location and number of the involved joints, with placement into the highest category possible based on the pattern of joint } \\
\text { involvement. 'Large joints' refers to shoulders, elbows, hips, knees and ankles. 'Small joints' refers to the MTP joints, proximal } \\
\text { interphalangeal joints, second to fifth MTP joints, thumb interphalangeal joints and wrists. 'Symmetric' is defined as bilateral } \\
\text { involvement of at least one region. In the category '>10 joints,' at least one of the involved joints must be a small joint; the other } \\
\text { joints can include any combination of large and additional small joints, as well as other joints not specifically listed elsewhere (eg, } \\
\text { temporomandibular, acromioclavicular, sternoclavicular, etc). } \\
\text { †Negative refers to international unit (IU) values that are less than or equal to the upper limit of normal (ULN) for the laboratory and } \\
\text { assay; low-positive refers to IU values that are higher than the ULN but three of less times the ULN for the laboratory and assay; } \\
\text { high-positive refers to IU values that are more than three times the ULN for the laboratory and assay. When rheumatoid factor (RF) } \\
\text { information is only available as positive or negative, a positive result should be scored as low-positive for RF. } \\
\text { łNormal/abnormal is determined by local laboratory standards. } \\
\text { §Duration of symptoms refers to patient self-report of the duration of signs or symptoms of synovitis (eg, pain, swelling, tenderness) } \\
\text { of joints that are clinically involved at the time of assessment, regardless of treatment status. } \\
\text { ACPA, anti-citrullinated protein antibody; CRP, C-reactive protein; ESR, erythrocyte sedimentation rate. }\end{array}$} \\
\hline
\end{tabular}

probability of developing RA. A 2-day workshop was held in May 2009 in which domains (factors) and categories within those domains that were important in determining the probability of developing RA were identified. When appropriate, these judgements were informed by the results of Phase 1 and other available literature. The relative importance or weights of these domains and their categories were determined by means of decision science theory and conjoint adaptive technology, using the computerised 1000Minds program (http:// www.1000minds.com) in an interactive and iterative process. ${ }^{26}$ This analysis permitted the calculation of an individual's score of the likelihood of developing RA from 0 to 100, in which a higher score indicated greater likelihood of RA development. The domains, categories and weights derived during that initial process are shown in table 2 .

\section{OBJECTIVES, METHODS AND RESULTS OF PHASE 3 Objectives of Phase 3}

In Phase 3 the working group integrated the findings of the first two phases, refined the scoring system, and determined the optimal cut point to define 'definite RA'. The goal of this final phase was to utilise the results of Phases 1 and 2 to develop a scoring system that would be applicable to newly presenting patients with undifferentiated inflammatory arthritis to permit identification of those with a high probability of developing persistent and/or erosive RA. Being intended for use with newly presenting patients, the scoring system should be robust enough that it could be applied repeatedly during the early course of disease, such that a patient identified as not classifiable as having definite RA at initial presentation might be classified as having definite RA at a subsequent time point. The work was not aimed at classifying individuals with established disease, either active or inactive. However, the working group recognised that patients may present for the first time with disease that is at a later stage and being treated. Thus, although it was not the explicit charge of the working group to provide rules for the classification of such patients, it is appropriate to have a single criteria system that could be applied to all patients; these issues were addressed by the expert panel during Phase 3 .

\section{Determination of the optimal cut point for definite RA}

Determination of the optimal cut point to classify an individual as having definite RA was achieved using two complementary approaches, mirroring the approaches used in the first two phases: data informed and consensus based. From the consensus-based approach, the expert panel was asked to examine the rankings of case scenarios based on the new scoring system and to indicate, in their opinion, the point at which the cases changed from 'probable' to 'definite' RA. Four cases were excluded due to missing domain information $(\mathrm{n}=2)$ or ineligibility (two cases were more likely another diagnosis). For the remaining 50 cases, the mean cut point defining definite RA was 65.7 (median 66.1; range 60.0-70.3) of a total possible score of 100 .

A data-driven verification of that cut point was then attempted, in which the new scoring system was applied to three of the existing cohorts used for Phase 1 (the Etude et Suivi des Polyarthrites Indifferenciees Recentes dataset from France, the Norwegian dataset and the Rotterdam Early Arthritis Cohort dataset from Rotterdam). ${ }^{25}$ These cohorts were chosen based on the completeness of data and the collected variables, enabling calculation of the patients' probability scores at baseline. The disease characteristics of these cohorts were not substantively different from those of the remaining cohorts (data not shown). 
The area under the curve (AUC) for the three receiver operating characteristic curves (which plot sensitivity against 1 -specificity for the range of scores) indicated good discrimination of those who did versus those who did not receive methotrexate (or another DMARD/biological agent) within a year (AUC 0.82 for Norway, 0.66 for France, and AUC 0.69 for Rotterdam; $\mathrm{p}<0.0001$ for all). The probability scores similarly discriminated between those who fulfilled the 1987 ACR criteria at 12 months and those who did not (AUC for the receiver operating characteristic curves 0.88 (Norway), 0.67 (France) and 0.72 (Rotterdam)). Visual inspection of the diagnostic test parameters associated with curves that used methotrexate initiation as the outcome showed a maximum slope for both the positive and negative likelihood ratios between a score of $60 / 100$ and 70/100, with flattening thereafter (67 in the Norway cohort, 66 in the French cohort and 66 in the Rotterdam cohort). The cut point of 60-70 that was derived from expert consensus was therefore supported by these data. Given the consistency with the consensus-based approach, and to maximise sensitivity of the criteria, a cut point of 60 was deemed to be most appropriate.

\section{Rationale for the composition and weight of the final criteria}

For development of the final criteria set, the results and weights from the comprehensive Phase 2 process ${ }^{26}$ were used as a starting point. Based on these categories and weights, we aimed in the final steps of the project to simplify the criteria in order to ensure that they were user friendly. We used the results of the data-driven Phase 1 as a guide for these adaptations, and verified at each step that the main properties of the criteria were not altered and that classification of patients remained unchanged.

The general steps towards simplification are shown in table 2, and included rescaling the scoring system to a scale of $0-10$. Then, each of the category weights was rounded to multiples of 0.5 . We tested the rounded scoring system in the case scenarios that had been used in Phase 2, and found no changes in the ranking of the cases compared with the exact scale. According to the rescaling, the cut point for definite RA would be $6 / 10$ or greater.

Despite their slightly different weights, the categories of asymmetric and symmetric oligoarthritis of large joints were merged for several reasons: first, symmetry was not found to be significantly important in the data analysis during Phase $1,{ }^{25}$ and second, the impact of merging was minimal when all possible presentations of patients with symmetric or asymmetric large joint inflammation were explored. For simplicity and ease of use, an integer scale was sought for all components of the scoring system. Thus, the high positive serology category was rounded from 3.5 (originally 3.39) to an integer of 3 because in no instance would classification status be altered by this change. In addition, the weight for abnormal acute-phase response was rounded from 0.5 (originally 0.59 ) to 1 , based on the stronger weight of acute-phase response (and of high-level acute-phase response) in Phase $1^{25}$

\section{Validation of the final criteria set}

The final criteria set with its simplified scoring system was further validated. Using the Phase 2 patient case scenarios, the correlation between cases' mean derived probability scores (0-100) and the proportion of expert panel members who indicated that they would initiate treatment with methotrexate out of concern about risk for persistence and/or erosive damage was strong
(Spearman's $r=0.82, \mathrm{p}<0.0001)$. The correlation of score with the proportion of experts who would refer the patient to a trial of a new biological agent with inherent risks was similarly strong (Spearman's $\mathrm{r}=0.0 .85, \mathrm{p}<0.0001$ ).

As a further validation, three cohorts that were not used in the identification of factors from Phase 1 were studied (Leiden (The Netherlands), Leeds (UK) and Toronto (Canada)); their characteristics were not substantively different from those of the remaining cohorts. ${ }^{25}$ Among cohort participants who received methotrexate within a year from symptom onset, the proportions with a score of $6 / 10$ or greater were $96.8 \%$ (Leiden), $90.5 \%$ (Leeds) and $87.2 \%$ (Toronto).

Table 3 The 2010 American College of Rheumatology/European League Against Rheumatism classification criteria for RA

Target population (Who should be tested?): Patients who

1) have at least 1 joint with definite clinical synovitis (swelling)*

2) with the synovitis not better explained by another disease $\dagger$

Classification criteria for RA (score-based algorithm: add score of categories A-D; a score of $\geq 6 / 10$ is needed for classification of a patient as having definite $R A) \ddagger$

A. Joint involvement§

1 large joint

$2-10$ large joints

$1-3$ small joints (with or without involvement of large joints) ${ }^{* *}$

$4-10$ small joints (with or without involvement of large joints)

$>10$ joints (at least 1 small joint) $\dagger \dagger$

B. Serology (at least 1 test result is needed for classification) $\ddagger \ddagger$ Negative RF and negative ACPA

Low-positive RF or low-positive ACPA

High-positive RF or high-positive ACPA

C. Acute-phase reactants (at least 1 test result is needed for classification)§§ Normal CRP and normal ESR 0

Abnormal CRP or normal ESR 1

D. Duration of symptoms $\uparrow$ ศ

$<6$ weeks

$\geq 6$ weeks

"The criteria are aimed at classification of newly presenting patients. In addition patients with erosive disease typical of rheumatoid arthritis (RA) with a history compatible with prior fulfilment of the 2010 criteria should be classified as having RA. Patients with long-standing disease, including those whose disease is inactive (with or without treatment) who, based on retrospectively available data, have previously fulfilled the 2010 criteria should be classified as having RA.

tDifferential diagnoses differ in patients with different presentations, but may include conditions such as systemic lupus erythematosus, psoriatic arthritis and gout. If it is unclear about the relevant differential diagnoses to consider, an expert rheumatologist should be consulted.

‡Although patients with a score of less than $6 / 10$ are not classifiable as having RA, their status can be reassessed and the criteria might be fulfilled cumulatively over time. $\S$ Joint involvement refers to any swollen or tender joint on examination, which may be confirmed by imaging evidence of synovitis. Distal interphalangeal joints, first carpometacarpal joints and first metatarsophalangeal joints are excluded from assessment. Categories of joint distribution are classified according to the location and number of involved joints, with placement into the highest category possible based on the pattern of joint involvement.

I'Large joints' refers to shoulders, elbows, hips, knees and ankles.

**'Small joints' refers to the metacarpophalangeal joints, proximal interphalangeal joints, second to fifth metatarsophalangeal joints, thumb interphalangeal joints and wrists.

t+In this category, at least one of the involved joints must be a small joint; the other joints can include any combination of large and additional small joints, as well as other joints not specifically listed elsewhere (eg, temporomandibular, acromioclavicular, sternoclavicular, etc.).

$¥ \ddagger$ Negative refers to international unit (IU) values that are less than or equal to the upper limit of normal (ULN) for the laboratory and assay; low-positive refers to IU values that are higher than the ULN but three of less times the ULN for the laboratory and assay; high-positive refers to IU values that are more than three times the ULN for the laboratory and assay. When rheumatoid factor (RF) information is only available as positive or negative, a positive result should be scored as low-positive for RF. $\S \S$ Normal/abnormal is determined by local laboratory standards.

ๆศाDuration of symptoms refers to patient self-report of the duration of signs or symptoms of synovitis (eg, pain, swelling, tenderness) of joints that are clinically involved at the time of assessment, regardless of treatment status.

ACPA, anti-citrullinated protein antibody; CRP, C-reactive protein; ESR, erythrocyte sedimentation rate. 


\section{Eligibility for testing with the new criteria}

The classification criteria can be applied to any patient or otherwise healthy individual, as long as two mandatory requirements are met: first, there must be evidence of currently active clinical synovitis (ie, swelling) in at least one joint as determined by an expert assessor (table 3). All joints of a full joint count may be assessed for this purpose with the exception of the distal interphalangeal (DIP) joints, the first metatarsophalangeal (MTP) joint and the first carpometacarpal (CMC) joint, since these joints are typically involved in osteoarthritis. Although currently no methods other than clinical examination should be used to evaluate the presence of synovitis in this determination of eligibility, this may change in the future as validated imaging techniques become more widely available. Second, the criteria may be applied only to those patients in whom the observed synovitis is not better explained by another diagnosis (table 3). For example, conditions that should be considered and excluded include systemic lupus erythematosus, psoriatic arthritis and gout, among others.

\section{Classification criteria for RA}

Four additional criteria can then be applied to eligible patients, as defined above, to identify those with 'definite RA' these are shown in table 3. Application of these criteria provides a score of $0-10$, with a score of 6 or greater being indicative of the presence of definite RA. This final scoring system was derived from both Phase 1 and Phase 2 data. A patient with a score below 6 cannot be classified as having definite RA, but might fulfil the criteria at a later time point. Figure 1 depicts a tree algorithm that incorporates the weights of each domain and the cut point of 6 for classification as definite RA. To classify a patient as having or not having definite RA, a history of symptom duration, a thorough joint evaluation, and at least one serological test (RF or ACPA) and one acute-phase response measure (erythrocyte sedimentation rate (ESR) or C-reactive protein (CRP)) must be obtained. It is acknowledged that an individual patient may meet the definition of RA without requiring that all tests be performed. For example, patients with a sufficient number of joints involved and longer duration of symptoms will achieve six points regardless of their serological or acute-phase response status. However, for the purposes of clinical research and trial enrolment, documentation of each domain will be necessary for phenotyping.

\section{Other clinical presentations: erosions and late disease}

Because the aim of the new classification criteria is to enable diagnosis and treatment earlier in the course of disease to prevent disease complications, erosions were not considered for inclusion in the scoring system. However, as stated above, the working group recognised that patients may present at later stages of disease. In addition, a single criteria system that could be applied to all patients was desired. Therefore, in addition to those who are newly presenting, three other groups of patients had to be considered: (1) those with erosions typical of RA were deemed to have prima facie evidence of RA and can be classified as such; (2) those with longstanding disease, either active inactive, who, based on retrospectively available data, can be determined to have previously satisfied the classification criteria

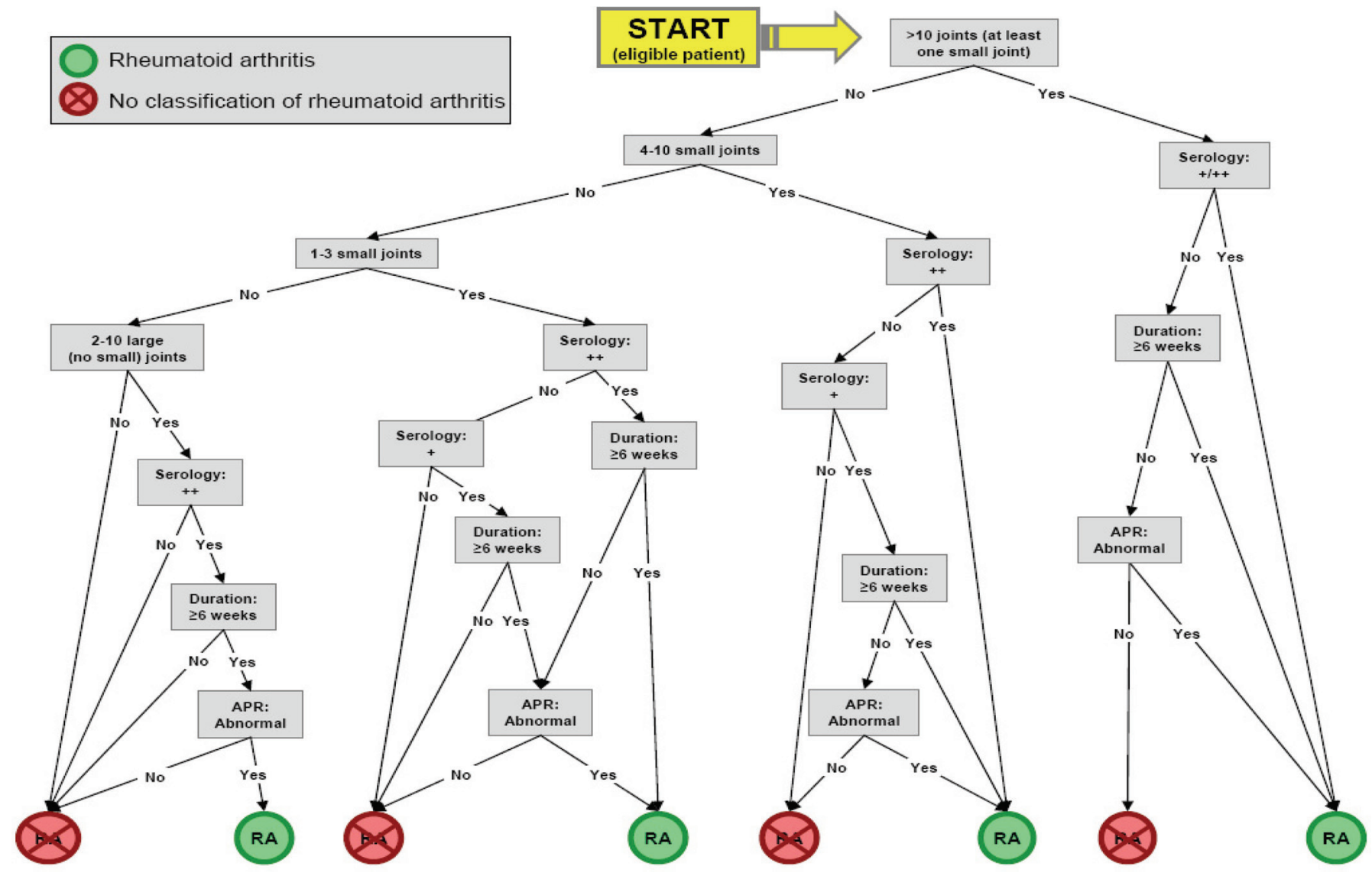

Figure 1 Tree algorithm to classify definite rheumatoid arthritis (RA) (green circles) or to exclude its current presence (red circles) among those who are eligible to be assessed by the new criteria. APR, acute-phase response. See footnotes to table 3 for definitions of categories (eg, serology + or ++ , or joint regions). 
can similarly be classified as having definite RA; and (3) in the setting of early disease that is being treated, individuals may not fulfil the new criteria at initial presentation, but may do so as their condition evolves over time.

\section{GLOSSARY OF DEFINITIONS}

In this section, we provide the detailed definitions necessary correctly and accurately to apply the new classification criteria for RA. A brief version of the glossary is included in the footnotes to table 3 .

\section{Definition of an 'involved' joint}

Joint involvement, as used for the determination of the pattern of joint distribution, differs from the definition of synovitis in one joint needed for eligibility in the eligibility criteria (see above): here it refers to any joint with swelling or tenderness on examination that is indicative of active synovitis. Tenderness is included as an equally important feature as swelling for the determination of joint involvement, particularly for the second to fifth MTP joints, in order to maximise sensitivity. Again, the DIP joints, the first MTP joint and the first CMC joint should not be considered, given their prevalent involvement in osteoarthritis. Furthermore, any joints with known recent injury that could contribute to swelling or tenderness should not be considered. Additional evidence of joint activity from other imaging techniques (such as MRI or ultrasound) may be used for confirmation of the clinical findings.

\section{Definition of small joints}

Small joints include the MTP, proximal interphalangeal, second to fifth MTP and thumb interphalangeal joints and the wrists. They do not include the first CMC, first MTP, or DIP joints, which are often affected by osteoarthritis.

\section{Definition of large joints}

The term 'large joints' refers to the shoulders, elbows, hips, knees and ankles.

\section{Determination of the joint pattern category}

Patients are categorised according to the number and location of involved joints by placing them into the category with the highest possible score. For example, a patient with involvement of two large joints and two small joints is placed in the category 'one to three small joints', as this category has the higher score. Patients should be scored for their joint involvement assuming that all of the peripheral joints indicated above have been assessed. For the highest category of joint involvement, in which $>10$ joints must be involved (including at least one small joint), additional joints that can be considered for inclusion in this count include the temporomandibular joint, sternoclavicular joint, acromioclavicular joint and others that may reasonably be expected to be involved in RA.

\section{Definition of the serological categories}

ACPA and IgM-RF levels are usually reported in IU. Based on the upper limit of normal (ULN) for the respective laboratory test and assay the following definitions can be made: negative=less than or equal to the ULN for the laboratory test and assay; lowlevel positive=higher than the ULN but $\leq 3$ times the ULN for the laboratory test and assay; high-level positive $=>3$ times the ULN for the laboratory test and assay. When RF information is available only qualitatively or as a level, and thus positive or negative, patients with a positive level should be scored as 'low-level positive' for RF. When a value for a serological test is not available or the normal range is not available for the reported test value, the result for that test should be considered 'negative/normal'. Patients should be scored only if information from at least one serological test is available.

\section{Definition of abnormal acute-phase response}

The acute-phase response measures CRP or ESR are scored as normal or abnormal based on the local laboratory standards. If results of at least one of these two tests are abnormal, the patient should be scored as having an abnormal acute-phase response. If a value for an acute-phase reactant is not available or information on the normal range for the reported test value is not available, the result of that test should be considered 'negative/normal'. For ESR, a standard approach that considers age and sex differences would be valuable. Patients should be scored only if at least one acute-phase response test is available for scoring.

\section{Definition of duration of symptoms}

The 'duration of symptoms' domain refers to the patient's selfreport of the maximum duration of signs or symptoms of synovitis (pain, swelling and tenderness) of any joint that is clinically involved at the time of assessment (ie, the day the criteria are applied). Thus, joints that are reported to have been previously symptomatic but are not involved at the time of assessment, whether due to treatment or not, should not be considered in estimating symptom duration.

\section{DISCUSSION}

We present here new classification criteria for RA, representing the culmination of an international collaborative effort supported by both a data-driven and a consensus-based approach. This classification scheme is designed to present a standardised approach to identifying that subset of individuals who present with an otherwise unexplained inflammatory arthritis of a peripheral joint(s), for whom the risk of symptom persistence or structural damage is sufficient to be considered for intervention with DMARD. This is thus the new proposed paradigm for the entity ' $R A$ ', importantly, not criteria for 'early' RA. If there was an intervention that was both infinitely effective and safe and could be provided at no cost and no discomfort, then there would be no requirement for such a subset to be identified, as every patient with inflammatory arthritis would be treated. Given that such an intervention does not exist, the search for appropriate classification rules is justified, and will also be helpful in guiding clinical diagnosis.

It is important, however, to stress that the criteria are meant to be applied only to eligible patients, in whom the presence of obvious clinical synovitis in at least one joint is central. They should not be applied to patients with mere arthralgia or to normal individuals. However, once definite clinical synovitis has been determined (or historical documentation of such has been obtained), as indicated in the glossary, a more liberal approach is allowed for determining the number and distribution of involved joints, which permits the inclusion of tender or swollen joints.

Symmetry is not a feature of the new criteria since it did not carry an independent weight in any phase of the work. In practice, symmetry is difficult to operationalise. Inevitably, though, the greater the number of involved joints the higher the likelihood of bilateral involvement. 
The development of these new criteria was based on diverse study cohorts and was performed by RA experts of diverse nationalities, enhancing the criteria set's generalisability. The final criteria also reflect consistency in domains of importance through the use of two independent methodologies. Previous classification schemes centered on a pre-identified clinical concept, typified by the occurrence of a predominantly symmetric small joint polyarthritis associated with autoantibody production and a high prevalence of erosions. ${ }^{27}$ This was the 'gold standard' used to devise the rules that could be applied in a repeatable manner to identify homogeneous groups for observation and study. The new criteria redefine RA, reflecting our collective hope that in the future, RA will no longer be characterised by erosive joint disease and persistence of symptoms, although these characteristics will continue to define established or longstanding untreated disease. This reflects several conceptual issues that are relevant in all areas of research and also in clinical practice.

Once the disease entity is redefined, existing epidemiological data on prevalence will have less relevance. Generally, this should not be a major concern, since there are well recognised difficulties in gathering and interpreting epidemiological data regarding the occurrence of RA: prevalence estimates are influenced by the effects of therapy and therefore are inherently unstable. The prevalence of RA could variously be described as the proportion of the population who have satisfied the new criteria at some relevant point in time.

The greater problem is the extrapolation of current literature on clinical trials and the design of future trials. The working group recommends that clinical trials should henceforth apply these new criteria; however, results from studies using the new criteria cannot necessarily be directly compared with the extensive body of existing work. In reality, in most trials, even of recent-onset RA, subjects with much higher levels of disease activity than is needed for fulfilment of the new criteria, and often those who have been treated unsuccessfully with multiple previous therapies, are selected. ${ }^{28}$ Thus, comparison between trials will be based much more on the distribution of disease activity at entry, for example, than the assumption that criteria satisfaction leads to homogeneity in patients recruited. Still, it might be useful, over this transition to the new criteria, for researchers to document the proportions of study subjects who fulfil the previous1987 and the new RA classification criteria, to enable comparisons.

There is a potential problem related to the pursuit of basic research. For example, genetic association studies have relied on a standardised approach to phenotypic assessment based on the 1987 ACR criteria. Such association studies still hold and, as stated above, the new criteria are likely to be easily satisfied by the participants in such studies. RA is always considered a heterogeneous disorder, and the new criteria scheme will probably increase that heterogeneity. Thus, basic scientists should be aware and, when appropriate, restrict recruitment or stratify results based on clinically meaningful phenotypes. For example, even among patients fulfilling the 1987 criteria, those who are ACPA positive and those who are ACPA negative have been shown to differ from a pathogenetic, clinical and prognostic perspective. ${ }^{29}$

The criteria have been intentionally derived from paper patient cases and cohorts of newly presenting individuals with undifferentiated inflammatory synovitis. Once classified, unless an alternative explanation for the synovitis becomes apparent over time, the individual is labelled as having 'definite RA'. However, acknowledging that RA is not a static disease, the new criteria have been developed such that they can be applied to patients at more than one time point in the evolution of their symptoms and signs. Thus, a patient who does not fulfil criteria for definite $\mathrm{RA}$ at first presentation might be classified as having definite RA at a subsequent time point.

Rather than developing a parallel system for 'established' disease or continuing to use the 1987 criteria for that purpose, the working group recommends that, when patient records allow, application of the criteria and assignment as definite RA may be made retrospectively. If there is a history compatible with definite RA as defined by these new criteria but no records of such, significant erosive disease seen on radiographs, typical of destructive RA, can be used as prima facie evidence of RA, precluding the need for applying additional criteria. Such individuals would need to be included as part of the total population of individuals affected by RA. It was not part of the working group's mission to define what is meant by significant erosive disease either in terms of the size, site, or number of erosions. Such agreement could be the task for further consensus, although current evidence suggests that such a definition should be highly specific. ${ }^{30}$ Thus, future work will be needed to define what evidence of erosions is acceptable to be considered as 'typical' of RA. This was a similar issue faced with the 1987 ACR criteria, but it did not detract from the usability of those criteria.

The use of the new criteria should be limited to a target population in whom there is otherwise no explanation (ie, definite diagnosis) for their synovitis. The working group has deliberately not provided an exhaustive list of diagnoses or tests that should be performed to exclude these, since that is not the purpose of classification criteria. Differential diagnosis is a physician responsibility and will be influenced by patient age and sex, practice type, as well as variations in the background population in terms of the incidence of 'competing' disorders. Thus, Lyme arthritis may be a frequent cause of synovitis in endemic areas, but testing for Borrelia burgdorferi would not be appropriate elsewhere. Differential diagnosis is, in this context, inevitably subject to differences in interpretation, as best exemplified by the conflict between those who would exclude patients with psoriasis from further classification. In this respect, the important utility of appropriate exclusion assessment is the avoidance of misclassifying patients as having RA who might otherwise not need to be treated or have self-limiting disease.

It was not the charge of the working group to create a referral tool for primary care physicians. Indeed, the original 1987 ACR criteria were also not designed for such use. Primary care physicians and other specialists need an easy-to-use tool to facilitate identification of individuals who have an inflammatory arthritic syndrome and for whom referral to a rheumatologist for further evaluation and diagnosis is appropriate. Support for such an endeavour is already being undertaken as a joint effort by ACR EULAR and other important stakeholders.

One limitation of the new criteria is that they are based on current knowledge. Genetic, proteomic, serological, or imaging biomarkers that provide a more robust basis for risk stratification may emerge, and this would necessarily lead to a modification or amendment of the 2010 criteria. Similarly, biomarkers, including imaging modalities, that more robustly identify highrisk subgroups of patients with synovitis may one day be available and validated. A pertinent example of a new biomarker is ACPA (typically, with testing for anti-cyclic citrullinated peptide). Based on a detailed literature review ${ }^{31}$ and our analytical approach to physician decision-making, ACPA status did not add importantly to the ability to classify an individual as having RA, beyond the information provided by RF when it is 
positive. The working group has therefore included both markers (ACPA and RF) equally in the criteria. However, as ACPA testing becomes more standardised, further refinement may be needed.

Because there is no gold standard for a diagnosis of RA, the cut-off score of 6 or greater is the best estimate from the current approaches used; testing in other cohorts will provide further evidence regarding its validity. Since the classification scheme actually provides a continuum of 'risk for developing persistent and/or erosive RA' (ie, it assigns the risk or probability of developing RA on a continuous score (from 0 to $100 \%$ )), there is scope for investigators to use other cut points-or multiple cut points-for different purposes. For example, in a clinical trial of a new potentially toxic agent, a higher, more conservative, cut point might be more appropriate; this is akin to clinical trials enrolling patients who meet criteria but also have evidence of a certain degree of severity or extent of involvement. In contrast, a population study of familial aggregation might use a less restrictive cut point. As such, there is information derived from scores across the range from 0 to 10 that may be utilised for different purposes in the future.

The working group has deliberately labelled these criteria as 'classification criteria' as opposed to 'diagnostic criteria'. The aim is to provide a standardised approach for discriminating, from a population of individuals presenting with undifferentiated synovitis, the subgroup with the highest probability of persistent or erosive RA, who may be enrolled into clinical trials and other studies through the use of uniform criteria. These individuals are also the ones who may therefore benefit from DMARD intervention. The criteria do not remove the onus on individual physicians, especially in the face of unusual presentations, to reach a diagnostic opinion that might be at variance from the assignment obtained using the criteria. Nonetheless, it is recognised that the new criteria will likely also be used as a diagnostic aid and be required to be satisfied, for example, by healthcare providers to enable access to particular interventions. However, much like other classification criteria, clinicians may be able to diagnose an individual who has not met the classification criteria definition or who has features that are not a component of the classification criteria. Diseases often present a much wider spectrum in clinical medicine that can be expected to be captured by classification criteria, the purpose of which is simply to provide a uniform set of standards by which an individual can be classified as having a clinical entity or not.

The new criteria need to be tested in several clinical situations and settings. Physicians need to report particularly if there is an important proportion of newly presenting patients who do not satisfy these criteria but for whom there is a compelling reason to treat with a DMARD, or who on follow-up, without a change in their classification status develop persistent or erosive disease. Validation in three of the cohorts available to us showed that the criteria were satisfied in $87-97 \%$ of the patients in whom the physicians chose to initiate methotrexate treatment.

In summary, the new ACR/EULAR classification criteria for RA present a new approach with a specific emphasis on identifying patients with a relatively short duration of symptoms who may benefit from early institution of DMARD therapy or entry into clinical trials of promising new agents that may halt the development of disease that currently fulfils the 1987 ACR criteria.

Acknowledgements The authors are grateful to Celina Alves, Carly Cheng, Tracey Farragher, Elisabeth Hensor, Jolanda Luime, Klaus Machold, Maria Dahl
Mjaavatten, Valerie Nell, Nathalie Rincheval, Marleen van de Sande and Annette van der Helm-van Mil, who were involved in development, data management, or maintenance of their respective datasets used in Phase 1 of the project, and to Rohit Aggarwal, Dinesh Khanna, Katherine Liao, Raj Nair and Sarah Ringold, who were involved in the design or implementation of Phase 2. The authors also wish to thank the ACR and the EULAR for their financial support of this project. The authors are especially grateful to Amy Miller and Regina Parker from the ACR and Heinz Marchesi and Anja Schönbächler from the EULAR, for their administrative support of the project.

Funding Dr. Aletaha has received consulting fees, speaking fees, and/or honoraria from Abbott, Bristol-Myers Squibb, UCB, Schering-Plough, Wyeth, and Roche (less than $\$ 10,000$ each). Dr. Bingham has received consulting fees, speaking fees, and/or honoraria from UCB, Roche, Genentech, Celgene, and Merck Serono (less than $\$ 10,000$ each); he has received research and/or educational grant support from Bristol-Myers Squibb, Genentech, UCB, Centocor, Abbott, and Amgen. Dr. Birnbaum has received consulting fees, speaking fees, and/ or honoraria from Amgen, Pfizer, Centocor, Abbott, and UCB (less than $\$ 10,000$ each). Dr. Burmester has received consulting fees, speaking fees, and/or honoraria from Abbott, Bristol-Myers Squibb, Pfizer, UCB, and Roche (less than $\$ 10,000$ each). Dr. Bykerk has received consulting fees, speaking fees, and/or honoraria from Amgen, Wyeth, Abbott, Schering-Plough, Roche, Bristol-Myers Squibb, and UCB (less than $\$ 10,000$ each); her spouse is employed by Genzyme and owns stock in the company. Dr. Cohen has received consulting fees, speaking fees, and/ or honoraria from UCB, Genentech, Bristol-Myers Squibb, and Human Genome Sciences (less than $\$ 10,000$ each). Dr. Combe has received consulting fees, speaking fees, and/or honoraria from Abbott, Bristol-Myers Squibb, Pfizer, Roche, Schering-Plough, and Merck, Sharpe, and Dohme (less than $\$ 10,000$ each). Dr. Emery has received consulting fees, speaking fees, and/or honoraria from Pfizer, Abbott, Centocor, UCB, Roche, Bristol-Myers Squibb, and Merck, Sharpe, and Dohme (less than $\$ 10,000$ each). Dr. Ferraccioli holds a patent for T cell receptor clonotype analysis (PCT/IB 2008/053152 NP). Dr. Huizinga has received consulting fees, speaking fees, and/or honoraria from Schering-Plough, BristolMyers Squibb, UCB, Biotest AG, Wyeth/Pfizer, Novartis, Roche, Sanofi-Aventis, Abbott, and Axis-Shield (less than $\$ 10,000$ each). Dr. Kavanaugh has conducted clinical research for Amgen, Abbott, Bristol-Myers Squibb, UCB, Roche, Centocor, Genentech, and Sanofi-Aventis. Dr. Kay has received consulting fees from Array BioPharma, Bristol-Myers Squibb, Celgene, Centocor, Genentech, Roche, UCB, and Sanofi-Aventis (less than $\$ 10,000$ each). Dr. Mease has received consulting fees, speaking fees, and/or honoraria from Abbott, Amgen, Biogen Idec, Bristol-Myers Squibb, Centocor, Roche, Genentech, UCB, Pfizer, Novartis, and Eli Lilly lless than $\$ 10,000$ each). Dr. Ménard has received unrestricted educational and research grants as well as consulting and speaking fees from Abbott, Amgen, Inova, Merck, Pfizer, Roche, Schering-Plough, UCB, and Wyeth (less than \$10,000 each) and investigator-initiated research grants from Bristol-Myers Squibb, Eurolmmun AG, and Roche (more than $\$ 10,000$ each); he owns stock or stock options in Merck; and he has a license agreement with Eurolmmun AG for an anti-Sa enzyme-linked immunosorbent assay. Dr. Moreland has received consulting fees, speaking fees, and/or honoraria from Biogen Idec, Centocor, Pfizer, Takeda, KaloBios, ChemoCentryx, UCB, Genentech, Incyte, and Eli Lilly (less than $\$ 10,000$ each). Dr. Naden has received consulting fees from the American College of Rheumatology in regard to the methodology of developing weighted scoring systems (more than $\$ 10,000)$. Dr. Pincus has received consulting fees, speaking fees, and/or honoraria from Amgen, Abbott, Bristol-Myers Squibb, Centocor, UCB, Wyeth, and Genentech (less than $\$ 10,000$ each) and investigator-initiated research grants from Amgen, Bristol-Myers Squibb, UCB, and Centocor. Dr. Stanislawska-Biernat has received speaking fees from Abbott and Pfizer (less than $\$ 10,000$ each). Dr. Vencovský has received speaking fees from Pfizer, UCB, Abbott, Roche, and Merck, Sharpe, and Dohme (less than $\$ 10,000$ each)

\section{Competing interests None.}

Contributors All authors were involved in drafting the article or revising it critically for important intellectual content, and all authors approved the final version to be published. Dr Silman had full access to all of the data in the study and takes responsibility for the integrity of the data and the accuracy of the data analysis. Study conception and design. Aletaha, Neogi, Silman, Felson, Birnbaum, Bykerk Combe, Costenbader, Dougados, Emery, Hazes, Huizinga, Kay, Kvien, Moreland, Naden, Smolen, Stanislawska-Biernat, Vencovský, Hawker.

Acquisition of data. Aletaha, Neogi, Silman, Bingham, Birnbaum, Burmester, Bykerk, Combe, Costenbader, Dougados, Emery, Hazes, Huizinga, Kavanaugh, Kay, Kvien, Laing, Ménard, Naden, Smolen, Stanislawska-Biernat, Tak, Upchurch, Vencovský, Hawker.

Analysis and interpretation of data. Aletaha, Neogi, Silman, Funovits, Bingham, Birnbaum, Burmester, Bykerk, Cohen, Combe, Dougados, Emery, Ferraccioli, Hazes, Hobbs, Huizinga, Kay, Laing, Mease, Ménard, Moreland, Naden, Pincus, Smolen, Stanislawska-Biernat, Symmons, Tak, Upchurch, Vencovský, Wolfe, Hawker. .

Provenance and peer review Not commissioned; externally peer reviewed. 
Author affiliations ${ }^{1}$ Medical University of Vienna, Vienna, Austria

${ }^{2}$ Boston University School of Medicine, Boston, Massachusetts, USA

${ }^{3}$ Arthritis Research UK, Chesterfield, UK

${ }^{4} J o h n s$ Hopkins University, Baltimore, Maryland, USA

${ }^{5}$ California Pacific Medical Center and University of California, San Francisco,

California, USA

${ }^{6}$ Charité Hospital, University Medicine Berlin, Free University and Humboldt University

Berlin, Berlin, Germany

${ }^{7}$ Mount Sinai Hospital and University of Toronto, Toronto, Ontario, Canada

${ }^{8}$ National Jewish Medical and Research Center, Denver, Colorado, USA

${ }^{9}$ Lapeyronie Hospital and Montpellier I University, Montpellier, France

${ }^{10}$ Brigham and Women's Hospital and Harvard University, Boston, Massachusetts,

USA

${ }^{11}$ Cochin Hospital, Assistance Publique-Hôpitaux de Paris and Paris-Descartes

University, Paris, France

12University of Leeds and NIHR Leeds Muscoskeletal Biomedical Research Unit Leeds, UK

${ }^{13}$ School of Medicine, Catholic University of the Sacred Heart, Rome, Italy

${ }^{14}$ Erasmus Medical Center, University Medical Center Rotterdam and University of

Rotterdam, Rotterdam, The Netherlands

${ }^{15}$ University of Colorado School of Medicine, Denver, Colorado, USA

${ }^{16}$ Leiden University Medical Centre, Leiden, The Netherlands

${ }^{17}$ University of California, San Diego, California, USA

${ }^{18}$ UMass Memorial Medical Center and University of Massachusetts Medical

School,Worcester, Massachusetts, USA

${ }^{19}$ Diakonhjemmet Hospital, Oslo, Norway

20University of Michigan, Ann Arbor, Michigan, USA

${ }^{21}$ Swedish Medical Center and University of Washington, Seattle, Washington, USA

${ }^{22}$ McGill University Health Centre and McGill University, Montreal, Quebec, Canada

${ }^{23}$ University of Pittsburgh, Pittsburgh, Pennsylvania, USA

${ }^{24}$ Ministry of Health, Auckland, New Zealand

${ }^{25}$ New York University Hospital for Joint Diseases, New York, New York, USA

${ }^{26}$ Institute of Rheumatology, Warsaw, Poland

${ }^{27}$ University of Manchester, Manchester, UK

${ }^{28}$ Academic Medical Center, University of Amsterdam, Amsterdam, The Netherlands

${ }^{29}$ Institute of Rheumatology, Prague, Czech Republic

${ }^{30}$ National Data Bank for Rheumatic Diseases and University of Kansas, Wichita, Kansas, USA

${ }^{31}$ Women's College Hospital and University of Toronto, Toronto, Ontario, Canada

\section{REFERENCES}

1. Scott DL, Symmons DP, Coulton BL, et al. Long-term outcome of treating rheumatoid arthritis: results after 20 years. Lancet 1987;1:1108-11.

2. Mitchell DM, Spitz PW, Young DY, et al. Survival, prognosis, and causes of death in rheumatoid arthritis. Arthritis Rheum 1986;29:706-14.

3. Pincus T, Callahan LF, Sale WG, et al. Severe functional declines, work disability, and increased mortality in seventy-five rheumatoid arthritis patients studied over nine years. Arthritis Rheum 1984:27:864-72

4. Isomäki H. Long-term outcome of rheumatoid arthritis. Scand J Rheumatol Suppl 1992;95:3-8.

5. Wolfe F. The natural history of rheumatoid arthritis. J Rheumato/ Suppl 1996;44:13-22.

6. Aho K, Heliövaara M, Maatela J, et al. Rheumatoid factors antedating clinical rheumatoid arthritis. J Rheumatol 1991;18:1282-4.

7. Aho K, von Essen R, Kurki $\mathrm{P}$, et al. Antikeratin antibody and antiperinuclear factor as markers for subclinical rheumatoid disease process. J Rheumato/ 1993;20:1278-81.

8. Nielen MM, van Schaardenburg D, Reesink HW, et al. Specific autoantibodies precede the symptoms of rheumatoid arthritis: a study of serial measurements in blood donors. Arthritis Rheum 2004;50:380-6.
9. Rantapää-Dahlqvist $\mathbf{S}$, de Jong BA, Berglin E, et al. Antibodies against cyclic citrullinated peptide and $\lg A$ rheumatoid factor predict the development of rheumatoid arthritis. Arthritis Rheum 2003;48:2741-9.

10. Firestein GS. Evolving concepts of rheumatoid arthritis. Nature 2003;423:356-61.

11. Smolen JS, Aletaha D, Koeller M, et al. New therapies for treatment of rheumatoid arthritis. Lancet 2007;370:1861-74.

12. Bohndorf $\mathbf{K}$, Schalm J. Diagnostic radiography in rheumatoid arthritis: benefits and limitations. Baillieres Clin Rheumatol 1996;10:399-407.

13. Van der Heijde DM. Joint erosions and patients with early rheumatoid arthritis Br J Rheumatol 1995;34(Suppl 2):74-8.

14. Plant MJ, Jones PW, Saklatvala J, et al. Patterns of radiological progression in early rheumatoid arthritis: results of an 8 year prospective study. J Rheumatol 1998;25:417-26.

15. Wolfe F, Sharp JT. Radiographic outcome of recent-onset rheumatoid arthritis: a 19-year study of radiographic progression. Arthritis Rheum 1998;41:1571-82.

16. Machold KP, Stamm TA, Eberl GJ, et al. Very recent onset arthritis - clinical, laboratory, and radiological findings during the first year of disease. J Rheumatol 2002:29:2278-87.

17. Bijlsma JW, Weinblatt ME. Optimal use of methotrexate: the advantages of tight control. Ann Rheum Dis 2007;66:1409-10.

18. Pincus T, Yazici Y, Sokka T, et al. Methotrexate as the "anchor drug" for the treatment of early rheumatoid arthritis [review]. Clin Exp Rheumatol 2003;21 (Suppl 31):S179-85.

19. Visser K, van der Heijde D. Optimal dosage and route of administration of methotrexate in rheumatoid arthritis: a systematic review of the literature. Ann Rheum Dis 2009;68:1094-9.

20. Doan T, Massarotti E. Rheumatoid arthritis: an overview of new and emerging therapies. J Clin Pharmacol 2005;45:751-62.

21. van der Heide A, Jacobs JW, Bijlsma JW, et al. The effectiveness of early treatment with "second-line" antirheumatic drugs. A randomized, controlled trial. Ann Intern Med 1996;124:699-707.

22. Bukhari MA, Wiles NJ, Lunt M, et al. Influence of disease-modifying therapy on radiographic outcome in inflammatory polyarthritis at five years: results from a large observational inception study. Arthritis Rheum 2003;48:46-53.

23. van Dongen $\mathbf{H}$, van Aken J, Lard LR, et al. Efficacy of methotrexate treatment in patients with probable rheumatoid arthritis: a double-blind, randomized, placebocontrolled trial. Arthritis Rheum 2007;56:1424-32.

24. Arnett FC, Edworthy SM, Bloch DA, et al. The American Rheumatism Association 1987 revised criteria for the classification of rheumatoid arthritis. Arthritis Rheum 1988;31:315-24.

25. Funovits J, Aletaha D, Bykerk V, et al. The 2010 American College of Rheumatology/ European League Against Rheumatism classification criteria for rheumatoid arthritis: methodological report Phase I. Ann Rheum Dis 2010;69:1589-95.

26. Neogi T, Aletaha D, Silman AJ, et al. The 2010 American College of Rheumatology/ European League Against Rheumatism Classification Criteria for Rheumatoid Arthritis: Phase 2 methodological report. Arthritis Rheum 2010;62:2582-91.

27. Van der Helm-van Mil AH, Detert J, le Cessie S, et al. Validation of a prediction rule for disease outcome in patients with recent-onset undifferentiated arthritis: moving toward individualized treatment decision-making. Arthritis Rheum 2008:58:2241-7.

28. Hyrich KL, Symmons DP, Silman AJ.OMERACT 7 Special Interest Group. Reconciling subject differences in recruitment to clinical trials and clinical practice. $J$ Rheumatol 2005;32:2475-6.

29. Huizinga TW, Amos $\mathrm{Cl}$, van der Helm-van Mil AH, et al. Refining the complex rheumatoid arthritis phenotype based on specificity of the HLA-DRB1 shared epitope for antibodies to citrullinated proteins. Arthritis Rheum 2005;52:3433-8.

30. Thabet MM, Huizinga TW, van der Heijde DM, et al. The prognostic value of baseline erosions in undifferentiated arthritis. Arthritis Res Ther 2009;11:R155.

31. Aggarwal R, Liao K, Nair R, et al. Anti-citrullinated peptide antibody assays and their role in the diagnosis of rheumatoid arthritis. Arthritis Rheum 2009:61:1472-83. 
Corrections

doi:10.1136/ard.2010.138461corr1

Aletaha D, Neogi T, Silman A J, et al. 2010 Rheumatoid arthritis classification criteria: an American College of Rheumatology/European League Against Rheumatism collaborative initiative. Ann Rheum Dis 2010;69:1580-88. Table 3 was published with the incorrect header, missing letters $A, B, C$ and $D$ and missing scores. The full text and pdf have been corrected online. The authors have noted additional errors: the following should read: Abnormal CRP or abnormal ESR and not Abnormal CRP or normal ESR. In table 2, MTP has been mentioned twice. It should read: 'Small joints' refers to the MCP joints, proximal interphalangeal joints, second to fifth MTP joints, thumb interphalangeal joints and wrists. Also, on page 1583, the Spearman r should be " 0.85 " rather than "0.0.85". 